\title{
Composição e estrutura da regeneração natural em até nove anos após a extração de Eucalyptus grandis Hill ex. Maiden no Vale do Itajaí, SC
}

\author{
Composition and structure of natural regeneration in nine years after the \\ extraction of Eucalyptus grandis Hill ex. Maiden in Itajaí Valley, SC
}

\author{
Daiane Luchetta Ronchi ${ }^{\text {I }}$, Samara Welter Duarte ${ }^{\mathrm{II}}$, Lauri Amândio Schorn ${ }^{\mathrm{III}}$
}

\section{Resumo}

A regeneração natural é considerada uma das técnicas mais promissoras de restauração de áreas degradadas em função dos aspectos ecológicos, silviculturais e econômicos. O presente estudo teve como objetivo avaliar características da regeneração natural em diferentes idades de estabelecimento, em áreas de preservação permanente, após a extração de povoamentos de Eucalyptus grandis. Foi realizado o levantamento e análise florística da vegetação regenerante em áreas com idades de regeneração natural de cinco e meio, sete e nove anos, ao longo das margens de um curso de água. Foram implantadas 56 parcelas amostrais de $10 \mathrm{~m} \times 20 \mathrm{~m}$ para o levantamento do estrato arbóreo e o mesmo número de subunidades para o levantamento do estrato regenerativo. Foi realizada a análise florística, por área e estrato da vegetação, e a comparação entre os três ambientes quanto à riqueza, diversidade, distribuição de grupos ecológicos e síndromes de dispersão. A vegetação estudada mostrou alterações na composição florística ao longo de nove anos. O número e a densidade de espécies pioneiras sofreram decréscimos, tanto no estrato arbóreo quanto no regenerativo, enquanto espécies clímax tolerantes à sombra tiveram acréscimos com o avanço das idades de regeneração. As espécies clímax exigentes em luz foram predominantes nos três ambientes, assim como a síndrome de dispersão zoocórica. A diversidade para as três áreas analisadas, e para os dois estratos, foi crescente com as idades de regeneração. Os ambientes com sete e nove anos de regeneração apresentaram maior porcentagem de espécies comuns, além de densidade e distribuição de grupos ecológicos mais semelhantes em relação ao ambiente com cinco anos e meio de regeneração.

Palavras-chave: Regeneração natural; Dinâmica sucessional; Síndromes de dispersão

\begin{abstract}
Natural regeneration is considered one of the most promising techniques for restoration of degraded areas due to ecological, silvicultural and economic aspects. The present study aimed to evaluate the characteristics of natural regeneration at different ages of establishment, in permanent preservation areas, after Eucalyptus grandis stands extraction. A survey and floristic analysis of the regenerating vegetation was performed in areas with natural regeneration ages of five and a half, seven and nine years, along the banks of a watercourse. Fifty-six sample plots of $10 \mathrm{~m}$ x $20 \mathrm{~m}$ were implanted for the tree stratum survey and the same number of subunits for the regenerative stratum survey. Floristic analysis was performed by area and vegetation stratum, and the comparison between the three environments for richness, diversity, distribution of ecological groups and dispersion syndromes. The vegetation studied showed floristic composition changes along nine years. The number and density of pioneer species suffered decrease in both the arboreal and regenerative stratum, while shadow-tolerant clímax species had increase as the regeneration ages progress. Light demanding climax species were prevalent in all three environments, as was zoochoric dispersal syndrome. The diversity for the three areas analyzed, and for both strata, was increasing with the regeneration ages. Environments with seven and nine years of regeneration presented higher percentage of common species, besides density and distribution of more similar ecological groups in relation to environment with five and a half years of regeneration.
\end{abstract}

Keywords: Natural regeneration; Successional dynamics; Dispersion syndromes

Bióloga, MSc., Pesquisadora autônoma, Rua São Paulo, 3.250, Itoupava Seca, CEP 89030-000, Blumenau (SC), Brasil. daianeronchi@hotmail.com (ORCID: 0000-0003-0216-3367)

Engenheira Florestal, MSc., Pesquisadora Autônoma, Rua São Paulo, 3.250, Itoupava Seca, CEP 89030-000, Blumenau (SC), Brasil. swduarte0@ gmail.com (ORCID: 0000-0003-0842-7124)

III Engenheiro Florestal, Dr., Professor do Programa de Pós-Graduação em Engenharia Florestal, Universidade Regional de Blumenau, Rua São Paulo, 3.250, Itoupava Seca, CEP 89030-000, Blumenau (SC), Brasil. lauri.schorn@gmail.com (ORCID: 0000-0003-3732-5354) 


\section{Introdução}

A Floresta Ombrófila Densa cobria originalmente 31\% do território Catarinense, contudo sofreu alterações drásticas, especialmente a fragmentação devido à intensa exploração dos recursos naturais, principalmente pelas atividades agropastoris e expansão urbana desordenada. Atualmente, estudos realizados pelo Inventário Florístico Florestal de Santa Catarina registraram uma cobertura remanescente de $40,1 \%$ da Floresta Ombrófila Densa no estado, sendo que $74 \%$ desse total se encontram em fragmentos com até 50 hectares (VIBRANS et al., 2012).

Considerando a extensão das áreas atualmente degradadas e a serem recuperadas, éaconselhável que os modelos de restauração sejam acessíveis e apresentem menor custo de implantação. Nesse sentido, as técnicas de restauração ecológica podem usar técnicas diversas, de acordo com o grau de degradação a que as áreas foram submetidas, mas todas visam acelerar a regeneração natural e restaurar a cobertura florestal natural com foco primário na regeneração de espécies nativas (CHAZDON, 2016). A mesma autora cita que os métodos de restauração variam grandemente em relação aos custos, aos níveis de biodiversidade que eles suportam e ao tempo necessário para o desenvolvimento da floresta.

A regeneração natural passiva, considerada uma das técnicas de restauração ecológica de áreas degradadas, apresenta como principal vantagem o custo reduzido (TRENTIN et al., 2018). No entanto, leva a taxas mais lentas de desenvolvimento da floresta (CHAZDON, 2016). Dependendo da conectividade da paisagem, a regeneração natural pode proporcionar o estabelecimento de alta diversidade de espécies compondo uma gama de estratos vegetais, que são reconstituídos por interações planta-animal, conduzindo o sistema gradativamente para estágios sucessionais mais avançados, sendo um ótimo indicador de restauração (RODRIGUES et al., 2009). A progressão de estágios dentro do processo de sucessão é a grande responsável por garantir o enriquecimento de espécies, o aumento em complexidade estrutural e funcional do ecossistema (CHADZON, 2012). A medida que a sucessão avança e o sub-bosque se torna mais úmido e sombreado, a abundância de espécies que não toleram sombreamento diminui no sub-bosque, enquanto aumenta a abundância de espécies de variadas formas de vida e que toleram sombra (MUNIZ-CASTRO; WILLIAMS-LINERA; MARTÍNEZ-RAMOS, 2012; CHAZDON, 2016).

A regeneração natural passiva tem sido adotada na recuperação de áreas consideradas de preservação permanentes, como é o caso de margens de rios e nascentes, após o uso dessas áreas para fins agropecuários ou silviculturais, visando a adequação à legislação, especialmente à Lei 12.651/2012. Essa lei prevê inclusive o uso consorciado de espécies exóticas em fases iniciais do processo de recuperação e com o intuito de favorecer a regeneração natural.

Poucos estudos têm abordado a regeneração natural em áreas de preservação permanentes após a extração de reflorestamentos homogêneos. Entre esses estudos pode-se citar Seubert et al. (2017), que avaliaram a regeneração natural em áreas com idades entre 1,5 a 5 anos de regeneração no Vale do Itajaí em Santa Catarina, após a extração de povoamento de Eucalyptus. Esses autores evidenciaram notáveis mudanças na densidade e na distribuição dos grupos na vegetação com o avanço da idade, caracterizados pela diminuição das espécies pioneiras e aumento de espécies tolerantes à sombra. Em outro trabalho realizado em áreas de preservação permanentes na região do planalto norte de Santa Catarina, após a extração de povoamentos de Pinus taeda, Schorn et al. (2016) observaram mudanças graduais na composição da vegetação regenerante, caracterizadas especialmente pela diminuição de espécies pioneiras e ingressos de espécies de outros grupos. Concluíram também que, em geral, a vegetação natural remanescente nessas áreas apresenta boa resiliência, tornando-se um fator de impulso para a recuperação das áreas de preservação permanentes após a extração de povoamentos.

Portanto, estudos detalhados sobre a composição florística e sua dinâmica em áreas em processo de regeneração são importantes para embasar iniciativas voltadas para proteger, enriquecer, recuperar ou reconstituir a vegetação natural, especialmente em áreas de preservação permanente. Diante do exposto, este estudo teve por objetivo avaliar a composição florística, a densidade e a diversidade da vegetação nos estratos arbóreo e regenerativo, em três áreas sob regeneração natural, com diferentes idades de abandono, após a extração de reflorestamento de Eucalyptus grandis Hill ex Maiden. 


\section{Materiais e métodos}

O estudo foi realizado no município de Brusque, região do Vale do Itajaí em Santa Catarina, em áreas que anteriormente eram manejadas com a cultura de Eucalyptus grandis e foram abandonadas em diferentes períodos com o intuito de regularizá-las como Áreas de Preservação Permanente. A propriedade está inserida na Bacia Hidrográfica do Rio Itajaí, subbacia do Rio Itajaí Mirim, numa altitude aproximada de 206 metros e próximo às coordenadas $27^{\circ} 02$ ’07” S e 485’ $40^{\prime \prime}$ O.

A geologia da região da área de estudo é formada pelo Complexo Metamórfico Brusque e o relevo varia de ondulado a forte ondulado nas encostas e suave ondulado nas depressões dos fundos de vales. Predomina na área de estudo o Argissolo Vermelho-Amarelo Álico de textura média argilosa (EMBRAPA, 2013).

Conforme a classificação de Köppen, o município de Brusque apresenta clima do tipo Cfa - Clima Subtropical mesotérmico úmido com verão quente. Sua temperatura média anual varia de $18^{\circ} \mathrm{C}$ a $20^{\circ} \mathrm{C}$ com precipitação total anual de 1500 a $1700 \mathrm{~mm}$ bem distribuídos durante todo o ano e umidade relativa anual entre 84 e 86\% (EPAGRI, 2002). A vegetação da região estudada está inserida no bioma Mata Atlântica, na região fitoecológica Floresta Ombrófila Densa Submontana (IBGE, 2012).

A coleta de dados foi realizada em áreas caracterizadas por diferentes idades de abandono, após a extração dos povoamentos de Eucalyptus grandis, denominadas: a) área em regeneração natural com idade de cinco anos e meio (RN5); b) área em regeneração natural com idade de sete anos (RN7); c) área em regeneração natural com idade de nove anos (RN9). O povoamento anteriormente extraído apresentava idade de nove anos e foi implantado, conduzido e explorado através de operações semimecanizadas.

A avaliação do estrato arbóreo foi realizada a partir de unidades amostrais (UA) de 200 $\mathrm{m}^{2}(20 \mathrm{~m} \times 10 \mathrm{~m})$, demarcadas paralelamente ao principal curso de água existente no interior do imóvel, sendo distribuída em 16 UA na área RN5 $\left(3.200 \mathrm{~m}^{2}\right)$, 21 UA na área RN7 $\left(4.200 \mathrm{~m}^{2}\right)$ e 19 UA na área RN9 $\left(3.800 \mathrm{~m}^{2}\right)$. As unidades amostrais foram locadas de forma sistemática, distanciadas entre si em 20 metros e a uma distância média de 15 metros do início do leito do curso de água.

Em cada UA foram registrados e identificados todos os indivíduos arbóreos e arbustivos com CAP (circunferência a altura do peito) igual ou superior a $5 \mathrm{~cm}$ e altura superior a $1,3 \mathrm{~m}$. Para avaliação do estrato regenerativo, foi demarcada uma subunidade de $20 \mathrm{~m}^{2}$ (10 m x $2 \mathrm{~m}$ ) dentro de cada UA do estrato arbóreo, onde foram mensuradas todas as espécies arbóreas e arbustivas com CAP inferior a $5 \mathrm{~cm}$ e altura igual ou superior a $0,50 \mathrm{~m}$.

Foi coletado material botânico fértil ou não para herborização e posterior identificação das espécies encontradas. O material botânico coletado foi identificado pela comparação com exsicatas existentes no Herbário Dr. Roberto Miguel Klein da FURB. As espécies foram agrupadas em famílias, de acordo com o sistema do Angiosperm Phylogeny Group IV (2016).

Os dados coletados foram processados pelo software Mata Nativa 4 (CIENTEC, 2016) onde foram determinados os valores da diversidade florística. Para tanto, foram utilizados os índices de Shannon $\left(H^{\prime}\right)$ e de equabilidade de Pielou $\left(J^{\prime}\right)$, descritos por Magurran (2013), determinados para cada área e para os estratos arbóreo e regenerativo. Para a comparação dos dados entre as três áreas foi aplicado o teste t de Hutcheson $(<0,010)$.

As espécies foram classificadas segundo o grupo ecológico, adotando-se a metodologia sugerida por Oliveira-Filho (1994), nas seguintes categorias: pioneiras (P), clímax exigente de luz (CL) e clímax tolerante à sombra (CS).

Em relação à síndrome de dispersão, as espécies foram classificadas conforme Stefanello et al. (2010), sendo agrupadas em três grandes categorias: zoocórica, anemocórica e autocórica. 


\section{Resultados e discussão}

Nas três áreas foram amostrados 5.048 indivíduos, distribuídos em 162 espécies, 95 gêneros e 42 famílias. As famílias com maior número de espécies foram Myrtaceae (19), Asteraceae, Fabaceae e Melastomataceae (15), Rubiaceae (10), Lauraceae e Solanaceae (nove), perfazendo um total de $56,8 \%$ das espécies identificadas (Tabela 1 ).

Essas famílias são comumente encontradas em estudos de regeneração e sucessão florestal, sendo que resultados semelhantes para Floresta Ombrófila Densa foram obtidos por Ruschel et al. (2009) e Seubert et al. (2017) ao analisarem o processo sucessional de dois diferentes estágios sucessionais secundários.

\section{Tabela 1 - Espécies arbóreo-arbustivas presentes nas áreas em processo de regeneração} natural após a extração de Eucalyptus grandis, em Brusque (SC)

Table 1 - Tree-shrub species present in natural regeneration process areas after extraction of Eucalyptus grandis, in Brusque (SC)

\begin{tabular}{|c|c|c|c|c|c|}
\hline Família/ Espécie & RN5 & RN7 & RN9 & GE & SD \\
\hline \multicolumn{6}{|l|}{ Acanthaceae } \\
\hline Justicia carnea Lindl. & & & $\mathrm{R}$ & $\mathrm{P}$ & Zoo \\
\hline \multicolumn{6}{|l|}{ Annonaceae } \\
\hline Annona cacans Warm. & & A & A & CL & Zoo \\
\hline Annona neosericea H.Rainer & A & A & A & CL & Zoo \\
\hline Annona sylvatica A. St.-Hil. & & AR & A & CL & Zoo \\
\hline Guatteria australis A.St.-Hil. & A & A & & $\mathrm{CL}$ & Zoo \\
\hline Xylopia brasiliensis Spreng. & A & AR & AR & $\mathrm{CL}$ & Zoo \\
\hline \multicolumn{6}{|l|}{ Apocynaceae } \\
\hline Tabernaemontana catharinensis DC. & A & $\mathrm{R}$ & AR & $\mathrm{CL}$ & Zoo \\
\hline \multicolumn{6}{|l|}{ Aquifoliaceae } \\
\hline Ilex dumosa Reissek & A & A & & $\mathrm{P}$ & Zoo \\
\hline Ilex theezans Mart. ex Reissek & $\mathrm{R}$ & A & & CL & Zoo \\
\hline \multicolumn{6}{|l|}{ Arecaceae } \\
\hline Bactris setosa Mart. & & & A & CS & Zoo \\
\hline Euterpe edulis Mart. & & AR & AR & CS & Zoo \\
\hline Syagrus romanzoffiana (Cham.) Glassman & A & A & AR & CL & Zoo \\
\hline \multicolumn{6}{|l|}{ Asteraceae } \\
\hline Austroeupatorium inulaefolium (Kunth) R.M.King \& H.Rob. & A & & AR & $\mathrm{P}$ & Ane \\
\hline Baccharis dracunculifolia DC. & AR & A & A & $\mathrm{P}$ & Ane \\
\hline Baccharis microdonta DC. & A & A & & $\mathrm{P}$ & Ane \\
\hline Baccharis punctulata DC. & & AR & & $\mathrm{P}$ & Ane \\
\hline Baccharis semiserrata DC. & AR & A & & $\mathrm{P}$ & Ane \\
\hline Baccharis serrulata (Lam.) Pers. & A & & & $\mathrm{P}$ & Ane \\
\hline
\end{tabular}


Tabela 1 - Continuação ...

Table 1 -Continuation ...

Asteraceae

Baccharis subdentata DC.

Piptocarpha angustifolia Dusén ex Malme

Piptocarpha axillaris (Less.) Baker

Symphyopappus compressus (Gardner) B.L.Rob.

\begin{tabular}{ccccc} 
& & A & P & Ane \\
& & A & P & Ane \\
& A & A & P & Ane \\
& AR & A & CL & Ane \\
& & A & P & Ane \\
& A & & P & Ane \\
& & A & P & Ane \\
& A & & P & Ane \\
AR & A & & P & Ane \\
\hline
\end{tabular}

Symphyopappus itatiayensis (Hieron.) R.M.King \& H.Rob.

Vernonanthura discolor (Spreng.) H.Rob.

Vernonanthura divaricata (Spreng.) H.Rob.

Vernonanthura puberula (Less.) H.Rob.

Vernonanthura tweediana (Baker) H.Rob.

AR AR AR CL Ane

\begin{tabular}{|c|c|c|c|c|c|}
\hline Jacaranda puberula Cham. & AR & AR & AR & CL & Ane \\
\hline Bixaceae & & & & & \\
\hline Bixa orellana $\mathrm{L}$. & A & & & CL & Zoo \\
\hline
\end{tabular}

Boraginaceae

Cordia silvestris Fresen.

A CL Zoo

Cannabaceae

Trema micrantha (L.) Blume

$\begin{array}{llll}\text { A } & \text { A } & \text { P } & \text { Zoo }\end{array}$

Celastraceae

Maytenus aquifolia Mart.

A CS Zoo

Maytenus gonoclada Mart.

A CS Zoo

Maytenus robusta Reissek

A CS Zoo

Elaeocarpaceae

Sloanea guianensis (Aubl.) Benth.

A AR A CS Zoo

Euphorbiaceae

Alchornea glandulosa Poepp. \& Endl.

AR AR AR P Zoo

Alchornea triplinervia (Spreng.) Müll.Arg.

AR A CL Zoo

Sapium glandulosum (L.) Morong

A

CL Zoo

Fabaceae

Centrolobium microchaete (Mart. ex Benth.) H.C.Lima

Dahlstedtia pentaphylla (Taub.) Burkart

Dalbergia brasiliensis Vogel

Dalbergia frutescens (Vell.) Britton

A CL Ane

Enterolobium contortisiliquum (Vell.) Morong

A

Inga marginata Willd.

CL Aut

AR AR CL Zoo 
Tabela 1 - Continuação ...

Table 1 - Continuation ...

Família/ Espécie

Fabaceae

Lonchocarpus campestris Mart. ex Benth.

Lonchocarpus guilleminianus (Tul.) Malme

Lonchocarpus muehlbergianus Hassl.

Machaerium stipitatum (DC.) Vogel

Mimosa bimucronata (DC.) Kuntze

Ormosia arborea (Vell.) Harms

Platymiscium floribundum Vogel

Schizolobium parahyba (Vell.) Blake

Senna macranthera (DC. ex Collad.) H.S.Irwin \& Barneby

\section{Lamiaceae}

Aegiphila integrifolia (Jacq.) Moldenke

Aegiphila obducta Vell.

Vitex megapotamica (Spreng.) Moldenke

Lauraceae

Cryptocarya moschata Nees \& Mart. ex Nees

Endlicheria paniculata (Spreng.) J.F.Macbr.

Licaria armeniaca (Nees) Kosterm.

Nectandra membranaceae (Sw.) Griseb.

Nectandra oppositifolia Nees

Ocotea dispersa (Nees \& Mart.) Mez

Ocotea glaziovii Mez

Ocotea puberula (Rich.) Nees

Ocotea silvestris Vattimo-Gil

Magnoliaceae

Magnolia ovata (A. St.-Hil.) Spreng.

A A AR CL Zoo

Malpighiaceae

Bunchosia maritima (Vell.) J.F.Macbr.

AR CS Zoo

Malvaceae

Triumfetta rhomboidea Jacq.

AR AR P Zoo

Melastomataceae

Leandra dasytricha (A.Gray) Cogn.

Leandra fragilis Cogn.

Leandra melastomoides Raddi

Leandra regnellii (Triana) Cogn.

A AR CL Ane

A AR CL Ane

A CL Ane

AR AR A P Aut

A $\quad$ CL Zoo

A CL Ane

A P Ane

AR P Aut

A A $A \quad$ P Zoo

A CL Zoo

A CL Zoo

$\begin{array}{ccccc} & \text { A } & & \text { CL } & \text { Zoo } \\ \text { A } & \text { A } & \text { AR } & \text { CL } & \text { Zoo } \\ & & \text { A } & \text { CS } & \text { Zoo } \\ \text { AR } & \text { A } & \text { AR } & \text { CL } & \text { Zoo } \\ \text { AR } & \text { AR } & \text { AR } & \text { CL } & \text { Zoo } \\ & \text { AR } & \text { A } & \text { CL } & \text { Zoo } \\ & & \text { A } & \text { CS } & \text { Zoo } \\ & \text { A } & \text { A } & \text { CL } & \text { Zoo } \\ & & \text { AR } & \text { CS } & \text { Zoo }\end{array}$


Tabela 1 - Continuação ...

Table 1 -Continuation ...

\begin{tabular}{|c|c|c|c|c|c|}
\hline Família/ Espécie & RN5 & RN7 & RN9 & GE & SD \\
\hline \multicolumn{6}{|l|}{ Melastomataceae } \\
\hline Leandra variabilis Raddi & & AR & & CL & Zoo \\
\hline Miconia cabucu Hoehne & AR & AR & AR & CL & Zoo \\
\hline Miconia cinerascens Miq. & & $\mathrm{R}$ & A & $\mathrm{P}$ & Zoo \\
\hline Miconia cinnamomifolia (DC.) Naudin & & AR & AR & $\mathrm{P}$ & Zoo \\
\hline Miconia latecrenata (DC.) Naudin & & & $\mathrm{R}$ & CL & Zoo \\
\hline Miconia pusilliflora (DC.) Naudin & & & AR & CL & Zoo \\
\hline Miconia valtheri Naudin & & A & A & CL & Zoo \\
\hline Ossaea amygdaloides (DC.) Triana & & & $\mathrm{R}$ & CL & Zoo \\
\hline Tibouchina urvilleana (DC.) Cogn. & AR & AR & A & $\mathrm{P}$ & Ane \\
\hline \multicolumn{6}{|l|}{ Meliaceae } \\
\hline Cabralea canjerana (Vell.) Mart. & & AR & AR & CL & Zoo \\
\hline Cedrela fissilis Vell. & & AR & A & CL & Ane \\
\hline Guarea macrophylla Vahl & & AR & AR & CL & Zoo \\
\hline Trichilia lepidota Mart. & A & A & $\mathrm{R}$ & CS & Zoo \\
\hline Trichilia pallens C. DC. & & & AR & CS & Zoo \\
\hline \multicolumn{6}{|l|}{ Monimiaceae } \\
\hline Mollinedia argyrogyna Perkins & & & A & CS & Zoo \\
\hline Mollinedia schottiana (Spreng.) Perkins & & AR & AR & CS & Zoo \\
\hline Mollinedia triflora (Spreng.) Tul. & & AR & AR & CS & Zoo \\
\hline
\end{tabular}

\section{Moraceae}

Brosimum glazioui Taub.

A CL Zoo

Ficus adhatodifolia Schott

A AR A CL Zoo

Ficus cestrifolia Schott

A CL Zoo

\section{Myristicaceae}

Virola bicuhyba (Schott) Warb.

AR AR CL Zoo

Myrtaceae

Calyptranthes grandifolia O.Berg

Calyptranthes lucida Mart. ex DC.

Calyptranthes strigipes O.Berg

Campomanesia reitziana $\mathrm{D}$. Legrand

Campomanesia xanthocarpa O.Berg

Eugenia brasiliensis Lam.

Eugenia cereja D.Legrand

Eugenia kleinii D.Legrand

$\begin{array}{ccccc} & & \text { A } & \text { CS } & \text { Zoo } \\ & \text { A } & \text { A } & \text { CS } & \text { Zoo } \\ \text { R } & \text { A } & \text { CS } & \text { Zoo } \\ & \text { AR } & \text { AR } & \text { CL } & \text { Zoo } \\ & & \text { A } & \text { CL } & \text { Zoo } \\ \text { A } & \text { A } & \text { CL } & \text { Zoo } \\ & \text { A } & \text { CS } & \text { Zoo } \\ & \text { A } & \text { CS } & \text { Zoo }\end{array}$


Tabela 1 - Continuação ...

Table 1 - Continuation ...

\begin{tabular}{|c|c|c|c|c|c|}
\hline Família/ Espécie & RN5 & RN7 & RN9 & GE & SD \\
\hline \multicolumn{6}{|l|}{ Myrtaceae } \\
\hline Eugenia uniflora $\mathbf{L}$. & A & AR & & CL & Zoo \\
\hline Eugenia verticillata (Vell.) Angely & & & AR & CS & Zoo \\
\hline Marlierea tomentosa Cambess. & & A & A & CL & Zoo \\
\hline Myrceugenia myrcioides (Cambess.) O. Berg & & & AR & CS & Zoo \\
\hline Myrcia pubipetala Miq & A & A & A & CL & Zoo \\
\hline Myrcia spectabilis DC. & & A & A & CL & Zoo \\
\hline Myrcia splendens (Sw.) DC. & AR & AR & AR & CL & Zoo \\
\hline Myrcia tijucensis Kiaersk. & & & A & CL & Zoo \\
\hline Plinia peruviana (Poir.) Govaerts & & A & & CL & Zoo \\
\hline Psidium cattleianum Sabine & A & AR & A & CL & Zoo \\
\hline Psidium guajava $\mathrm{L}$. & AR & A & $\mathrm{A}$ & CL & Zoo \\
\hline \multicolumn{6}{|l|}{ Nyctaginaceae } \\
\hline Guapira opposita (Vell.) Reitz & & AR & AR & CL & Zoo \\
\hline \multicolumn{6}{|l|}{ Peraceae } \\
\hline Pera glabrata (Schott) Poepp. ex Baill. & & A & A & CL & Zoo \\
\hline \multicolumn{6}{|l|}{ Phyllanthaceae } \\
\hline Hieronyma alchorneoides Allemão & & AR & A & CL & Zoo \\
\hline
\end{tabular}

Phytolaccaceae

Seguieria langsdorffii Moq.

A CS Zoo

\section{Piperaceae}

Piper aduncum L.

Piper amplum Kunth

Piper arboreum Aubl.

Piper cernuum Vell.

Piper crassinervium Kunth

Piper diospyrifolium Kunth

Piper malacophyllum (C.Presl) C.DC.

AR CL Zoo

Piper mosenii C.DC.

\begin{tabular}{|c|c|c|c|}
\hline \multicolumn{3}{|c|}{ AR } & \\
\hline \multirow{3}{*}{ A } & AR & AR & CL \\
\hline & AR & $\mathrm{AR}$ & CL \\
\hline & AR & $\mathrm{AR}$ & CL \\
\hline \multirow{3}{*}{ AR } & AR & AR & CL \\
\hline & AR & $\mathrm{R}$ & CL \\
\hline & & AR & CL \\
\hline AR & AR & $\mathrm{AR}$ & CL \\
\hline
\end{tabular}

Primulaceae

Quiina glaziovii Engl.

Rhamnaceae

Hovenia dulcis Thunb.

A

CL Zoo

Rosaceae

Eriobotrya japonica (Thunb.) Lindl.

A

CL Zoo 
Tabela 1 - Continuação ...

Table 1 -Continuation ...

Família/ Espécie

RN5 RN7 RN9 GE $\quad$ SD

Rubiaceae

Alseis floribunda Schott

Bathysa australis (A.St.-Hil.) K.Schum.

Margaritopsis astrellantha (Wernham) L. Andersson

Psychotria nemorosa Gardner

$\begin{array}{cccc} & \text { A } & \text { CS } & \text { Ane } \\ \text { AR } & \text { A } & \text { CS } & \text { Aut } \\ \text { AR } & \text { R } & \text { CS } & \text { Zoo } \\ & \text { AR } & \text { CS } & \text { Zoo } \\ & \text { AR } & \text { CL } & \text { Zoo } \\ \text { R } & \text { R } & \text { CS } & \text { Zoo } \\ \text { A } & \text { AR } & \text { CS } & \text { Zoo } \\ \text { A } & & \text { CL } & \text { Zoo } \\ & \text { A } & \text { CS } & \text { Zoo } \\ \text { R } & & \text { CS } & \text { Zoo }\end{array}$

Psychotria nuda (Cham. \& Schltdl.) Wawra

Psychotria officinalis (Aubl.) Raeusch. ex Sandwith

Psychotria suterella Müll.Arg.

Psychotria vellosiana Benth.

Rudgea jasminoides (Cham.) Müll. Arg.

Rudgea recurva Müll. Arg.

Rutaceae

Citrus cf. limon L.

A A AR CL Zoo

Zanthoxylum rhoifolium Lam.

A $\quad$ A $\quad$ A $\quad$ CL Zoo

Salicaceae

Casearia decandra Jacq.

A AR A CL Zoo

Casearia sylvestris $\mathrm{Sw}$.

A AR AR CL Zoo

\section{Sapindaceae}

Allophylus edulis (A.St.-Hil., Cambess. \& A. Juss.) Radlk.

A CL Zoo

Allophylus petiolulatus Radlk.

A A CS Zoo

Cupania vernalis Cambess.

A AR AR CL Zoo

Matayba intermedia Radlk.

AR AR AR CL Zoo

Sapotaceae

Chrysophyllum gonocarpum (Mart. \& Eichler) Engl.

AR

CL Zoo

Solanaceae

Aureliana wettsteiniana (Witasek) Hunz. \& Barbosa

A CL Zoo

Brugmansia suaveolens (Willd.) Bercht. \& J.Presl

Brunfelsia uniflora (Pohl) D.Don

Cestrum axillare Vell.

Cestrum intermedium Sendtn.

Solanum compressus L.B. Sm. \& Downs

Solanum mauritianum Scop.

Solanum pseudoquina A. St.-Hill.

AR

Solanum variabile Mart.

$\begin{array}{ccccc} & & \text { A } & \text { CL } & \text { Zoo } \\ & \text { A } & & \text { CL } & \text { Zoo } \\ & \text { R } & & \text { CL } & \text { Zoo } \\ & & \text { A } & \text { CS } & \text { Zoo } \\ & \text { R } & & \text { CL } & \text { Zoo } \\ & \text { A } & \text { A } & \text { CL } & \text { Zoo } \\ & \text { R } & & \text { P } & \text { Zoo } \\ \text { AR } & \text { A } & \text { A } & \text { P } & \text { Zoo } \\ & \text { A } & & \text { P } & \text { Zoo }\end{array}$

Ci. Fl., Santa Maria, v. 30, n. 2, p. 380-395, abr./jun. 2020 
Tabela 1 - Conclusão ...

Table 1 - Conclusion ...

\begin{tabular}{|c|c|c|c|c|c|}
\hline Família/ Espécie & RN5 & RN7 & RN9 & GE & SD \\
\hline \multicolumn{6}{|l|}{ Urticaceae } \\
\hline Cecropia glaziovii Snethl. & A & AR & AR & $\mathrm{P}$ & Zoo \\
\hline Boehmeria caudata Sw. & & AR & AR & $\mathrm{P}$ & Aut \\
\hline Urera nitida (Vell.) P.Brack & & & AR & $\mathrm{CL}$ & Zoo \\
\hline
\end{tabular}

Fonte: Autores (2019)

Em que: $\mathrm{A}$ = estrato arbóreo; $\mathrm{R}$ = estrato regenerativo; $\mathrm{GE}=$ grupo ecológico; $\mathrm{P}=$ pioneira; $\mathrm{CL}$ = clímax exigente em luz; $C S$ = clímax tolerante à sombra; $S D$ = síndrome de dispersão; Zoo = zoocórica; Ane = anemocórica e Aut = Autocórica.

Ainda na Floresta Ombrófila Densa, mas com transição com a Floresta Ombrófila Mista, Scariot e Reis (2010) encontraram famílias semelhantes ao estudarem a regeneração natural de corredores ciliares. Venzke e Martins (2013) citaram muitas dessas famílias ao descreverem a composição e as relações florísticas do componente arbóreo-arbustivo de três estágios sucessionais de mata ciliar de uma Floresta Estacional Semidecidual.

Nas unidades amostrais do estrato arbóreo, os indivíduos se distribuíram em 154 espécies e 41 famílias, resultando em uma densidade total de 3.940 indivíduos/ha. No estrato regenerativo, a densidade resultou em 5.669 indivíduos/ha, distribuídos em 95 espécies e 32 famílias.

As três áreas apresentaram 32 espécies comuns (19,75\%). Quando considerados separadamente os estratos, obteve-se $14,2 \%$ de espécies comuns no estrato arbóreo e apenas $4,3 \%$ no estrato regenerativo. Nesse aspecto, as áreas RN7 e RN9 mostraram-se mais semelhantes em relação à RN5, pois apresentaram $50,3 \%$ de espécies comuns, tendo $42,6 \%$ no estrato arbóreo superior e $17,9 \%$ no estrato regenerativo.

Em valores quantitativos, a área RN5, caracterizada por vegetação com menor tempo de regeneração, diferenciou-se das demais, especialmente da RN9, tanto no estrato arbóreo, quanto no estrato regenerativo, apresentando valores inferiores também para os parâmetros espécies, famílias, gêneros, número de indivíduos e densidade total (Tabela 2).

Tabela 2 - Número total de espécies, gêneros, famílias, indivíduos e densidade por hectare nos estratos arbóreo e regenerativo das áreas em processo de regeneração natural após a extração de Eucalyptus grandis, em Brusque (SC)

Table 2 - Total number of species, genera, families, individuals and density per hectare in the arboreal and regenerative strata of the natural regeneration process area after Eucalyptus grandis extraction in Brusque (SC)

\begin{tabular}{lccccccc}
\hline \multirow{2}{*}{ Parâmetros } & \multicolumn{3}{c}{ Estrato Arbóreo } & \multicolumn{3}{c}{ Estrato Regenerativo } \\
\cline { 2 - 7 } & RN5 & RN7 & RN9 & RN5 & RN7 & RN9 \\
\hline Espécies & $49 \mathrm{~B}$ & $102 \mathrm{AB}$ & $118 \mathrm{~A}$ & $22 \mathrm{~b}$ & $62 \mathrm{a}$ & $61 \mathrm{a}$ \\
\hline Gêneros & $39 \mathrm{~B}$ & $71 \mathrm{~A}$ & $74 \mathrm{~A}$ & $18 \mathrm{~b}$ & $48 \mathrm{a}$ & $42 \mathrm{a}$ \\
\hline Famílias & $25 \mathrm{~B}$ & $34 \mathrm{~A}$ & $35 \mathrm{~A}$ & $12 \mathrm{~b}$ & $27 \mathrm{a}$ & $26 \mathrm{a}$ \\
\hline No indivíduos & $404 \mathrm{~B}$ & $1.973 \mathrm{~A}$ & $2.036 \mathrm{~A}$ & $94 \mathrm{c}$ & $224 \mathrm{~b}$ & $317 \mathrm{a}$ \\
\hline Densidade (ind./ha) & $1.263 \mathrm{~B}$ & $4.698 \mathrm{~A}$ & $5.358 \mathrm{~A}$ & $2.938 \mathrm{c}$ & $5.333 \mathrm{~b}$ & $8.342 \mathrm{a}$ \\
\hline
\end{tabular}

Fonte: Autores (2019)

Em que: RN5 = área em regeneração natural com idade de cinco anos e meio; RN7 = área em regeneração natural com idade de sete anos; RN9 = área em regeneração natural com idade de nove anos. Médias seguidas pelas mesmas letras nas linhas, maiúsculas para o estrato arbóreo e minúsculas para o estrato regenerativo, não diferem estatisticamente pelo Teste de Tukey ao nível de $5 \%$ de probabilidade. 
Os números de espécies, de gêneros e de famílias foram crescentes com as idades de regeneração das áreas, es pecialmente no estrato arbóreo. O mesmo comportamento foi observado para a densidade, incluindo-se o estrato regenerativo (Tabela 2). Resultados semelhantes foram obtidos por Schorn e Galvão (2006) e Seubert et al. (2017) ao avaliarem a dinâmica da regeneração natural de indivíduos arbóreos em três estágios de sucessão de uma Floresta Ombrófila Densa Submontana e obtendo valores crescentes de densidade e riqueza com o avanço dos estágios sucessionais.

No estrato regenerativo, nas três áreas, foram encontradas 61 espécies e 26 famílias, sendo que as famílias mais representativas em número de espécies foram Melastomataceae (9), Piperaceae (7) e Rubiaceae (5). Piperaceae apresentou a maior densidade nesse estrato, com 2.500 indivíduos por hectare.

Em relação aos grupos ecológicos, 34 espécies foram classificadas como pioneiras, 95 como clímax exigentes de luz e 33 como clímax tolerante à sombra (Tabela 1).

$\mathrm{Na}$ área $\mathrm{RN} 5$, nos dois estratos amostrados, as pioneiras apresentaram o menor número de espécies, mas obtiveram o maior número de indivíduos. A predominância de espécies clímax exigentes em luz e pioneiras, bem como o menor número de espécies clímax tolerantes à sombra, comprova que a RN5 se encontra no início do processo de sucessão quando comparada com os outros ambientes estudados. No entanto, na área RN7, para os dois estratos amostrados, o maior valor de espécies pioneiras indica estágio intermediário, pois ainda se encontram espécies que foram importantes no estágio inicial. Já na área RN9, a predominância de espécies clímax exigentes de luz e clímax tolerantes à sombra indica que a vegetação se encontra em estágio mais avançado de sucessão. Em estudo realizado na mesma área, quatro anos antes, Seubert et al. (2017) encontraram uma distribuição semelhante de espécies em grupos ecológicos nas três áreas. Os resultados do presente trabalho e anteriores sugerem que, em curtos períodos de regeneração após o abandono de áreas antes ocupadas com reflorestamento de Eucalyptus, já é possível verificar o dinamismo da vegetação natural através da sucessão de espécies e de grupos ecológicos.

Nas três áreas, nos dois estratos analisados, foi predominante o grupo ecológico clímax exigentes em luz, que representou mais de $57 \%$ das espécies. Resultados semelhantes foram obtidos por Callegaro et al. (2012), ao avaliarem a regeneração natural de um fragmento de mata ciliar, e Seubert et al. (2017), em estudo anterior na mesma área deste trabalho. Paralelamente observou-se que, com o avanço da idade de regeneração, há um nítido decréscimo das espécies pioneiras e um aumento das espécies clímax tolerantes à sombra (Figura 1), confirmando que existe uma substituição gradual das categorias sucessionais no processo da sucessão florestal, reforçando as observações de Chazdon (2016), de que a regeneração de florestas tropicais segue um cenário geral de substituição de espécies intolerantes à sombra e de crescimento rápido por tolerantes à sombra de crescimento mais lento. Esse padrão é comum e citado nos estudos de Schorn e Galvão (2006), Pereira et al. (2010) e Souza et al. (2012). 
Figura 1 - Grupos ecológicos das espécies presente nos estratos arbóreo (A) e regenerativo (R) das áreas em processo de regeneração natural após a extração de Eucalyptus grandis, em Brusque (SC)

Figure 1 - Ecological groups of the species present in the arboreal (A) and regenerative (R) strata of the areas in the natural regeneration process after the extraction of Eucalyptus grandis, in Brusque (SC)

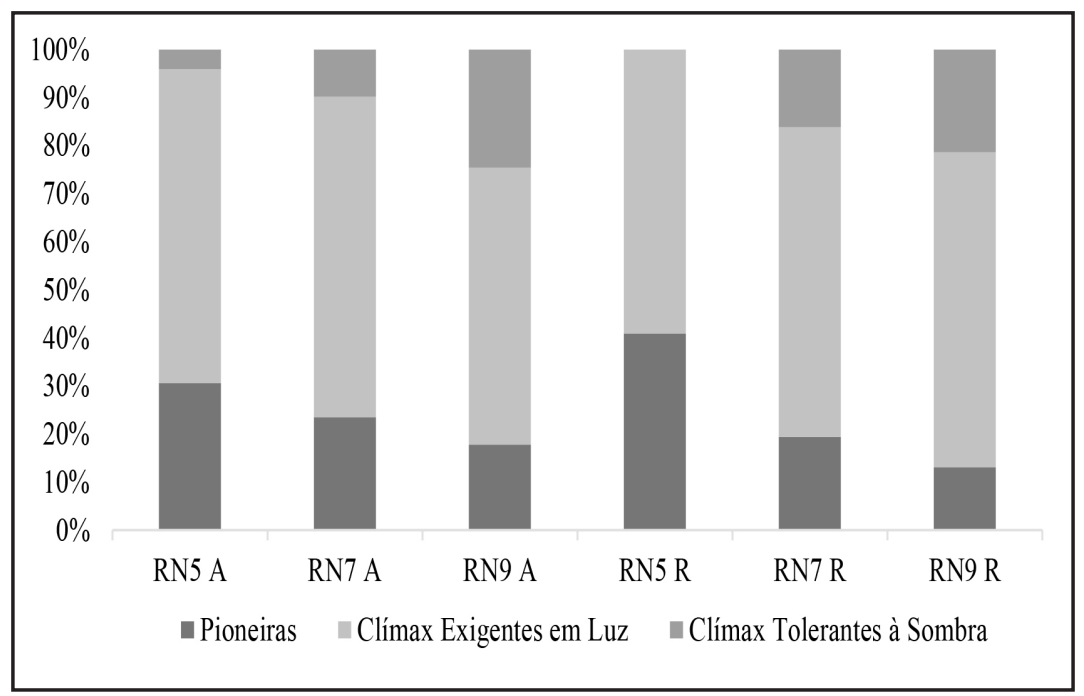

Fonte: Autores (2019)

Apesar da tendência observada, de diminuição de espécies pioneiras e aumento de espécies de outros grupos ecológicos com o avanço das idades da regeneração, quantidade considerável de espécies foram comuns nos ambientes em estudo, especialmente entre RN7 e RN9. Os resultados obtidos no presente trabalho em relação à distribuição dos grupos ecológicos de espécies evidenciam que os ambientes RN7 e RN9 podem apresentar características que permitem abrigar composições florísticas pouco distintas, possivelmente em função das pequenas diferenças de idades de regeneração e da proximidade entre as áreas. Esse fato também pode ser amparado pelas considerações de Chazdon (2016) de que as distinções clássicas entre pioneiras e não pioneiras proposta por Swaine e Whitmore (1988) é baseada nas respostas de sementes e plântulas e que não se aplicam claramente aos estágios de vida posteriores. Nessa mesma ótica, Wright (2010) mencionou que a maior parte das espécies lenhosas ocupa posições intermediárias no espectro entre espécies pioneiras e tolerantes à sombra.

Entre as espécies amostradas, a síndrome de dispersão predominante foi a zoocoria, representada por 127 espécies $(78,4 \%)$, seguida da anemocoria, com 29 espécies $(17,9 \%)$ e da autocoria com apenas 6 espécies (3,7\%). Segundo Scariot e Reis (2010), a predominância da zoocoria indica avanço do processo sucessional.

Em todas as áreas e estratos amostrados, observou-se que com o avanço da idade de regeneração houve um nítido decréscimo das espécies anemocóricas e um aumento das espécies zoocóricas. Além disso, que a proporção destas foi predominante nas três áreas, com índices acima de 65\% (Figura 2). Valores semelhantes foram obtidos nos estudos de Callegaro et al. (2012), Souza et al. (2012) e Venzke e Martins (2013), que encontraram percentuais variando de 56,8 a $82 \%$ de espécies zoocóricas. 
Figura 2 - Síndromes de dispersão das espécies presentes nos estratos arbóreo (A) e regenerativo $(\mathbf{R})$ das áreas em processo de regeneração natural após a extração de Eucalyptus grandis, em Brusque (SC)

Figure 2 - Dispersion syndromes of the present species in the arboreal (A) and regenerative (R) strata of the natural regeneration process after the extraction of Eucalyptus grandis, in Brusque

$(\mathrm{SC})$

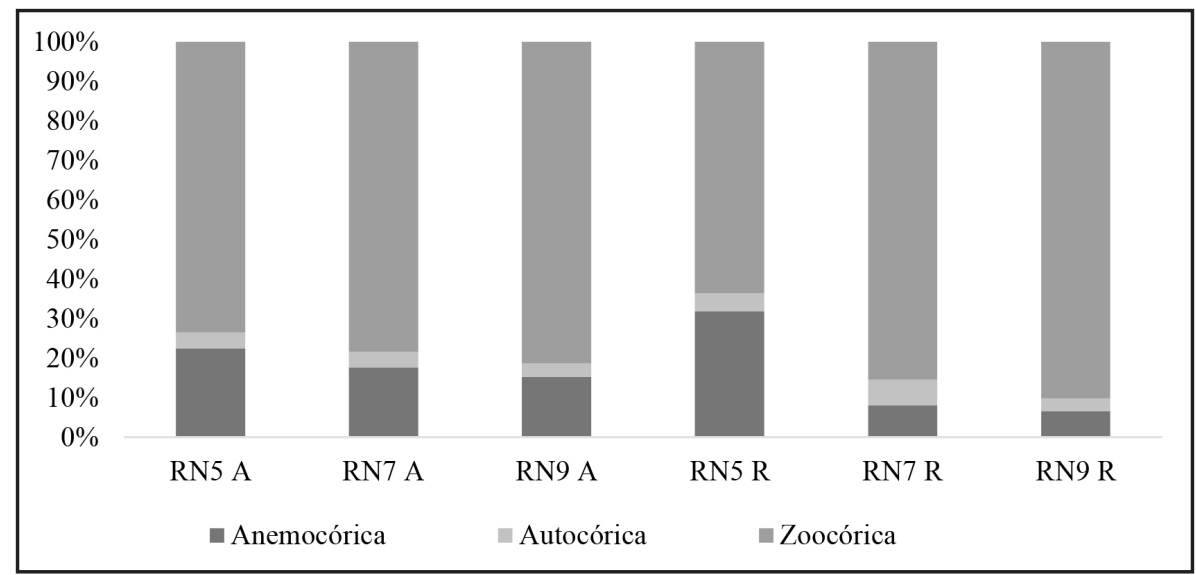

Fonte: Autores (2019)

De forma geral, apesar dos indivíduos das espécies zoocóricas serem predominantes em todas as áreas, não se observou a dominância de poucas, mas a contribuição do somatório de várias espécies para obtenção da representatividade. Desse modo, a presença dos agentes de dispersão é importante para a formação da estrutura das comunidades e populações florestais pois a distância de polinização e dispersão de sementes tem consequências sobre a composição genética das populações durante a regeneração florestal (CHAZDON, 2016). Isso porque o processo de dispersão de sementes significa a ligação entre a última fase reprodutiva da planta com a primeira fase do recrutamento da população (GALETTI et al., 2012) e a dispersão de sementes é fator essencial para a colonização de habitats e a constituição da estrutura espacial e temporal das populações de plantas (BARBOSA et al., 2009). No presente trabalho, a participação expressiva de espécies zoocóricas reforça o caráter dinâmico da regeneração, evidenciando o potencial de restauração de áreas de preservação permanentes através da regeneração natural, após a extração de povoamento de Eucalyptus.

A diversidade para as três áreas analisadas e para os dois estratos, igualmente foi crescente, aumentando conforme a idade de regeneração. No estrato arbóreo os valores variaram de 3,2 a 3,9 nats/ind., e no estrato regenerativo de 2,6 a 3,7 nats/ind (Tabela 3). Esse resultado evidencia a existência de relação entre a diversidade de espécies com a idade de regeneração.

Todos os resultados foram significativos (valor $\mathrm{p}<0,01$ ), indicando que as três áreas diferem quanto aos índices de Shannon e Pielou, tanto no estrato arbóreo quanto no estrato regenerativo. Os intervalos de confiança não se sobrepõem, dessa forma, quanto maior a lacuna entre os intervalos, mais diferentes são as áreas quanto à diversidade e equabilidade. 
Tabela 3 - Índice de diversidade de Shannon e equabilidade de Pielou nos estratos arbóreo e regenerativo das áreas em processo de regeneração natural após a extração de Eucalyptus grandis, em Brusque (SC)

Table 3 - Shannon diversity index and Pielou equability in the arbored and regenerative strata of the natural regeneration process after Eucalyptus grandis extraction in Brusque (SC)

\begin{tabular}{lccc}
\hline \multicolumn{1}{c}{ Parâmetros } & RN5 & RN7 & RN9 \\
\hline \multicolumn{4}{c}{ Estrato Arbóreo } \\
\hline Shannon $\left(\mathbf{H}^{\prime}\right)$ & $3,2 \pm 0,11(\mathrm{~A})$ & $3,6 \pm 0,05(\mathrm{~B})$ & $3,9 \pm 0,05(\mathrm{C})$ \\
Pielou $(\mathbf{J})$ & $0,81 \pm 0,02(\mathrm{~A})$ & $0,78 \pm 0,01(\mathrm{~B})$ & $0,81 \pm 0,01(\mathrm{C})$ \\
\hline \multicolumn{2}{c}{ Estrato Regenerativo } & $3,7 \pm 0,1(\mathrm{C})$ \\
\hline Shannon $\left(\mathbf{H}^{\prime}\right)$ & $2,6 \pm 0,2(\mathrm{~A})$ & $3,6 \pm 0,16(\mathrm{~B})$ & $0,89 \pm 0,02(\mathrm{C})$ \\
\hline Pielou $(\mathbf{J})$ & $0,84 \pm 0,05(\mathrm{~A})$ & $0,85 \pm 0,03(\mathrm{~B})$ & \\
\hline
\end{tabular}

Fonte: Autores (2019)

Em que: RN5 = área em regeneração natural com idade de cinco anos e meio; RN7 = área em regeneração natural com idade de sete anos; RN9 = área em regeneração natural com idade de nove anos.

Letras iguais maiúsculas na mesma linha não diferem entre si significativamente de acordo com o teste $t$ de Hutcheson $(\mathrm{p}<0,01)$.

De forma geral, o índice de Pielou, para os dois estratos, foi alto, pois os indivíduos mostraram-se amplamente distribuídos entre as espécies, tendendo a uma razão de abundância e riqueza (Tabela 3). Os valores do estrato regenerativo foram crescentes conforme a idade da regeneração, enquanto no estrato arbóreo as áreas RN5 e RN9 apresentaram valores iguais $(0,81)$, e superiores à área RN7 (0,78).

Em relação ao estudo anterior realizado na mesma área (SEUBERT et al., 2017), os valores de H' para o estrato arbóreo foram superiores, enquanto a equabilidade de Pielou mostrou poucas mudanças. Por outro lado, foram considerados inferiores ao encontrado por Lingner et al. (2013) na mesma região fitogeográfica, mas com predomínio de uma vegetação em estágio avançado de regeneração.

Para o estrato regenerativo, os valores de H’ encontrados nas três áreas são superiores aos encontrados nos estudos de Nóbrega et al. (2008) que, em levantamentos da regeneração natural em dois remanescentes naturais, obtiveram valores de $\mathrm{H}^{\prime}=1,9$ e 2,1 nats/ind., na vegetação de várzea do Rio Mogi-Guaçu, SP. Já no estudo anterior o estrato regenerativo mostrou maiores valores de H’e J na área RN7, com idade intermediária de regeneração, diferentemente do presente estudo onde se observam valores crescentes de diversidade e equabilidade com o avanço das idades de regeneração.

\section{Conclusão}

Os resultados apontam que a regeneração natural nas áreas em estudo evidenciou o dinamismo da vegetação e o potencial de recuperação de áreas de preservação permanentes através desse processo em áreas com características semelhantes.

Com o avanço da idade de regeneração, ocorreram alterações nítidas na distribuição das espécies em grupos ecológicos com o decréscimo das espécies pioneiras e o aumento das espécies clímax tolerantes à sombra.

Nas três áreas e nos dois estratos analisados, foi predominante o grupo ecológico das espécies clímax exigentes em luz, assim como a síndrome de dispersão zoocórica, fator determinante para a manutenção das comunidades arbóreas em longo prazo. 
A diversidade para as três áreas analisadas, e para os dois estratos, foi crescente, conforme as idades de regeneração, evidenciando a relação entre diversidade e a idade de regeneração.

As áreas com maiores períodos em regeneração mostraram maior semelhança em variáveis analisadas, evidenciando maior dinamismo da vegetação nos períodos iniciais de regeneração.

\section{Referências}

ANGIOSPERM PHYLOGENY GROUP (APG). An update of the Angiosperm Phylogeny Group classification for the orders and families of flowering plants: APG IV. Botanical Journal of the Linnean Society., [s.1.], v. 181, p. 1-20. 2016.

BARbosA, J. M.; EISENlOHR, P. V.; RODRIGUES, M. A.; BARBOSA, K. C. Ecologia da dispersão de sementes em Florestas Tropicais. In: MARTINS, S. V. (Org.) Ecologia de florestas tropicais do Brasil. Viçosa: Editora UFV, 2009. p. 52-73.

CALLEGARO, R. M; LONGHI, S. J.; BIALI, L. J.; EBLING, A.; ANDRZEJEWSKI, C.; BRANDÃO, C. Regeneração natural avançada de um fragmento de mata ciliar em Jaguari, RS, Brasil. Revista Brasileira de Ciências Agrárias, Recife, v. 7, n. 2, p. 315-321, abr./jun. 2012.

CHAZDON, R. L. Regeneração de florestas tropicais. Boletim do Museu Paraense Emilio Goeldi. Ciências Naturais, Belém, v. 7, p. 195-218, 2012.

CHAZDON, R. L. Renascimento de florestas. Regeneração na era do desmatamento. São Paulo: Oficina de Textos, 2016.

CIENTEC. Mata Nativa 4: Sistema para análise fitossociológica e elaboração de planos de inventários e planos de manejo de florestas nativas. Viçosa: CIENTEC, 2016.

EMBRAPA - Empresa Brasileira de Pesquisa Agropecuária. Sistema Brasileiro de Classificação de Solos. Rio de Janeiro: EMBRAPA Solos, 2013.

EPAGRI. Empresa de Pesquisa Agropecuária e Extensão Rural de Santa Catarina. Atlas climatológico de Santa Catarina. Florianópolis: EPAGRI, 2002.

GALETTI, M.; PIZO, M. A.; MORELLATO, L. P. C. Fenologia, frugivoria e dispersão de sementes. In: CULLEN Jr., L.; VALLADARES-PADUA, C. RUDRAN, R. (Org.). Métodos de estudo em biologia da conservação e manejo da vida silvestre. Curitiba: Ed. UFPR; Fundação O Boticário de Proteção a Natureza, 2012. p. 395-422.

IBGE - Instituto Brasileiro de Geografia e Estatística. Manual Técnico da Vegetação Brasileira. 2 ed. Rio de Janeiro: IBGE, 2012.

LINGNER, D. V.; SCHORN, L. A.; VIBRANS, A. C.; MEYER, L.; SEVEGNANI, L.; GASPER, A. L. de; SOBRAL, M. G.; KRU GER, A.; KLEMZ, G.; SCHMIDT, R.; JÚNIOR, C. A. Fitossociologia do componente arbóreo/arbustivo da Floresta Ombrófila Densa em Santa Catarina. In: VIBRANS, A. C.; SEVEGNANI, L.; GASPER, A. L. de; LINGNER, D. V. (Ed.). Inventário Florístico Florestal de Santa Catarina, Vol. IV, Floresta Ombrófila Densa. Blumenau: Edifurb, 2013. p. 159-200.

MAGURRAN, A. E. Medindo a diversidade biológica. Curitiba: Ed. UFPR, 2013.

MUNIZ-CASTRO; WILLIAMS-LINERA; MARTÍNEZ-RAMOS. Dispersal mode, shade tolerance, and phytogeografical affinity of três species during secundary succession intropical montane cloud forest. Plant Ecology, [s.1.], Springer Netherlands, v. 213, p. 339-353, 2012.

NÓBREGA, A. M. F. da; VALERI, S. V.; PAULA, R. C. de; SILVA, S. A. da. Regeneração natural em remanescentes florestais e áreas reflorestadas da várzea do Rio Mogi-Guaçu, Luiz Antônio SP. Revista Árvore, Viçosa, v. 32, n. 5, p. 909-920, 2008.

OLIVEIRA-FILHO, A. T. Estudos ecológicos da vegetação como subsídios para programas de 
revegetação com espécies nativas: uma proposta metodológica. Cerne, Lavras, v. 1, n. 1, p. 64-72, 1994.

PEREIRA, I. M.; BOTELHO, S. A.; VAN DER BERG, E.; et al. Caracterização ecológica de espécies arbóreas ocorrentes em ambientes de mata ciliar, como subsídio à recomposição de áreas alteradas nas cabeceiras do rio grande, Minas Gerais, Brasil. Ciência Florestal, Santa Maria, v. 20, n.2, p. 235-253, 2010.

RODRIGUES, R.R.; LIMA, R.A.F.; GANDOLFI, S.; NAVE, A.G. On the restoration of high diversity forests: 30 years of experiences in the Brazilian Atlantic Forest. Biological Conservation, [s.1.], ScienceDirect, v.142, n.6, p.1242-1251, 2009.

RUSCHEL, A. R.; MANTOVANI, M.; REIS, M. S. dos; NODARI, R. O. Caracterização e dinâmica de duas fases sucessionais em floresta secundária da Mata Atlântica. Revista Árvore, Viçosa, v. 33, n. 1, p. 101-115, 2009.

SCARIOT, E. C.; REIS, A. Riqueza e estrutura florística de corredores ciliares em regeneração natural no Planalto Norte Catarinense, Sul do Brasil. Perspectiva, Erechim, v. 34, n. 125, p. 53-65, mar. 2010.

SCHORN, L. A.; GALVÃO, F. Dinâmica da regeneração natural em três estágios sucessionais de uma Floresta Ombrófila Densa em Blumenau, SC. Floresta, Curitiba, v. 36, n. 1, p. 59-74, jan./ abr. 2006.

SCHORN, L. A.; NADOLNY, M. C.; RONCHI, D. L.; ADENESKY FILHO, E.; HOFFMANN, L. T.; LESSA, P. R.; KRIEGER, A. Restauração de áreas de preservação permanente após a colheita de Pinus tarda L. através da regeneração natural. In: SCHORN, L. A.; NADOLNY, M. C. (Org.) Resultados de Pesquisas Florestais na Renova Floresta. Blumenau: Edifurb, 2016. p. 13-53.

SEUBERT, R. C.; MAÇANEIRO, J. P.; SCHORN, L. A.; SEBOLD, D. C. Regeneração natural em diferentes períodos de abandono de áreas após extração de Eucalyptus grandis Hill ex Maiden, em argissolo vermelho-amarelo álico, em Brusque, Santa Catarina. Ciência Florestal, Santa Maria, v. 27 , n. 1, p. 1-19, mar. 2017.

SOUZA, L. M.; FARIA, L. A. V. B; BOTELHO, S. A.; FONTES, M. A. L.; FARIA, J. M. R. Potencial da regeneração natural como método de restauração do entorno de nascente perturbada. Cerne, Lavras, v. 18, n. 4, p. 565-576, out./dez. 2012.

STEFANELlO, D.; IVANAUSKAS, N. M.; MARTINS, S. V.; SILVA, E.; KUNZ, S. H.; Síndromes de dispersão de diásporos das espécies de trechos de vegetação ciliar do rio das Pacas, Querência - MT. Acta Amazônica, v. 40, p. 141-150, 2010.

SWAINE, M. D.; WHITMORE, T. C. On the definition of ecological species groups in tropical rain forest. Vegetatio, Dordrecht, v. 75, n. 1-2, p. 81-86, 1988.

WRIGHT, S. The future of tropical forests. Annals of the New York Academy of sciences, New York, v. 1195, p. 1-27, 2010.

TRENTIN, B. E.; ESTEVAN, D. A.; ROSSETTO, E. F. S.; GORENSTEIN, M. R.; BRIZOLA, G. P.; BECHARA, R. C. Restauração florestal na Mata Atlântica: passiva, nucleação e plantio de alta diversidade. Ciência Florestal, Santa Maria, v. 28, n. 1, p. 160-174, jan.- mar., 2018.

VENZKE, T. S.; MARTINS, S. V. Aspectos florísticos de três estágios sucessionais em mata ciliar em Arroio do Padre, extremo sul do Brasil. Floresta, Curitiba, v. 43, n. 2, p. 191-204, abr./ jun. 2013.

VIBRANS, A.; McROBERTS, M. E.; LINGNER, D. V.; NICOLETTI, A. L.; MOSER, P. Extensão original e atual da cobertura florestal de Santa Catarina. In: VIBRANS, A. C.; SEVEGNANI, L.; GASPER, A. L de; LINGNER, D. V. (eds.) Inventário Florístico Florestal de Santa Catarina, Vol. I, Diversidade e conservação dos remanescentes florestais. Blumenau, Edifurb, 2012. p. 65-76. 